\title{
A Modified Generalized Chaplygin Gas as the Unified Dark Matter-Dark Energy Revisited
}

\author{
Xue-Mei Deng \\ Purple Mountain Observatory, Chinese Academy of Sciences, Nanjing 210008, China \\ E-mail: xmd@pmo.ac.cn
}

\begin{abstract}
A modified generalized Chaplygin gas (MGCG) is considered as the unified dark matter-dark energy revisited. The character of MGCG is endued with the dual role, which behaves as matter at early times and as an quiessence dark energy at late times. The equation of state for MGCG is $p=-\alpha \rho /(1+\alpha)-\vartheta(z) \rho^{-\alpha} /(1+\alpha)$, where $\vartheta(z)=-\left[\rho_{0 c}(1+z)^{3}\right]^{(1+\alpha)}\left(1-\Omega_{0 B}\right)^{\alpha}\left\{\alpha \Omega_{0 D M}+\Omega_{0 D E}\left[\omega_{D E}+\alpha\left(1+\omega_{D E}\right)\right](1+\right.$ $\left.z)^{3 \omega_{D E}(1+\alpha)}\right\}$. Some cosmological quantities, such as the densities of different components of the universe $\Omega_{i}$ ( $i$ respectively denotes baryons, dark matter and dark energy) and the deceleration parameter $q$, are obtained. The present deceleration parameter $q_{0}$, the transition redshift $z_{T}$ and the redshift $z_{e q}$, which describes the epoch when the densities in dark matter and dark energy are equal, are also calculated. To distinguish MGCG from others, we then apply the Statefinder diagnostic. Later on, the parameters $\left(\alpha\right.$ and $\omega_{D E}$ ) of MGCG are constrained by combination of the sound speed $c_{s}^{2}$, the age of the universe $t_{0}$, the growth factor $m$ and the bias parameter $b$. It yields $\alpha=-3.07_{-4.98}^{+5.66} \times 10^{-2}$ and $\omega_{D E}=-1.05_{-0.11}^{+0.06}$. Through the analysis of the growth of density perturbations for MGCG, it is found that the energy will transfer from dark matter to dark energy which reach equal at $z_{e q} \sim 0.48$ and the density fluctuations start deviating from the linear behavior at $z \sim 0.25$ caused by the dominance of dark energy.

PACS numbers: 98.80.-k.
\end{abstract}

Keywords: Dark energy; Dark matter 


\section{Introduction}

As standardized candles, type Ia supernovae suggest that the universe is undergoing accelerating expansion[1]. The same evidence has been shown by cosmic microwave background (CMB)[2] and large scale structure (LSS) [3] observations. All these data indicate the dominant component of the universe is relatively smooth and has a large negative pressure. All kinds of alternative models stand up for explaining this exotic phenomena within relativistic theory of gravity, which include the energy of the quantum vacuum (such as cosmological constant $\Lambda[4,[5]$ ), the existence of another new scalar field (quintessence [6] which is possibly related to the inflaton, k-essence[7], tachyon [8], phantom [9], and quintom[10]), and the influence of unseen additional spatial dimensions predicted by string theory[11]. Instead of the presence of dark energy, the most interesting idea for solving this dark riddle is depend on a new aspect of gravity which is not accounted for general relativity[12]. Unfortunately, the current astronomical observations data still can not determine completely the nature of dark energy or decide its existence since this problem has puzzled us for a long time.

As one of plausible dynamical models for dark energy, Chaplygin gas (CG) and the generalized Chaplygin gas (GCG)[13], has created a lot of interest in recent times[14]. CG or GCG behaves like pressureless dust at early times and like a cosmological constant during very late times. The fact that the properties of $\mathrm{CG}$ and GCG interpolate between those of cold dark matter (CDM) and a $\Lambda$-term makes the models to provide a conceptual framework for a unified model of dark matter and dark energy. Besides, it is more worthy of note that the GCG model has been successfully confronted with various phenomenological tests such as high precision CMB data, supernovae data and gravitational lensing [15]. On the other hand, since observations such as SNe Ia[1] are still not sufficient to establish evidence of a dynamical equation of state for dark energy, we can naturally consider a fluid consisted of dark matter and quiessence dark energy with constant $\omega_{D E}$ which full of the whole universe. Thus, we propose a modified generalized Chaplygin gas (MGCG) as the unified dark matter and dark energy revisited on some previous works [18, 19, 20].

With future deeper and more intensive surveys of SNe Ia, such as Supernovae acceleration probe (SNAP) [29], it provides a chance to distinguish different models of dark energy so that we will consider the so-called "geometrical" or Statefinder diagnostic since it can probe the expansion dynamics of the universe through higher derivatives of the scale factor. So, we must constrain the parameters $\left(\alpha\right.$ and $\left.\omega_{D E}\right)$ of the MGCG at first. The plat of this paper is as follows. In Sec. 2, we give corresponding fundamental cosmology equations about MGCG. Subsequently, in Sec. 3, we consider Statefinder diagnostic of the MGCG. We then constrain the parameters of the MGCG by means of the sound speed $c_{s}^{2}$, the age of the universe $t_{0}$, the growth factor $m$ and the bias parameter $b$ in Sec. 4. Finally, conclusion and our results are outlined in Sec. 5. 


\section{Fundamental cosmology equations about MGCG}

\subsection{Motivation}

An interesting unified model for two dark sectors is the Chaplygin gas (CG) model[16] introduced with $p=-A / \rho$. Although this model has been very successful in explaining the SNe Ia data, it shows that CG model does not pass the tests connected with structure formation and observed strong oscillations of the matter power spectrum[17. This situation can be alleviated in the generalized Chaplygin gas (GCG) proposed with $p=-A / \rho^{\alpha}$. And the parameter $\alpha$ is rather severely constrained, i.e., $0 \leq \alpha<0.2$ at the $95 \%$ confidence level[13]. Since the difference between $\Lambda$ CDM and GCG models is so tiny and the equation of state of dark energy still cannot be determined exactly, a new generalized Chaplygin gas (NGCG) 20 proposed by considering that $A$ in GCG is a function of redshift $z$. Following this idea, we develop GCG-like models from another view. As a purely kinetic k-essence model with a constant potential, the tachyon fields can be considered as CG model [18]. Meanwhile the tachyon field can also act as a source of dark energy depending upon the form of the tachyon potential. Thus, the authors in Ref. [19] introduced an extended tachyon field (ETF) and then provided a modified Chaplygin gas (MCG) [19], in which the equation of state (EOS) has a more generalized form

$$
p=-\frac{\alpha}{1+\alpha} \rho-\frac{1}{1+\alpha} \frac{A}{\rho^{\alpha}},
$$

where $A$ and $\alpha$ are constants. As a kind of new attempt just like the extension from GCG to NGCG, we present a modified generalized Chaplygin gas (MGCG) through replacing $A$ in Eq. (1) with a function $\vartheta(z)$ of redshift $z$. The analysis and discussion of the matter power spectrum for MGCG will be investigated in our future work by comparing the model with observations.

\subsection{Deduction of some cosmological quantities and discussion}

Within the framework of Friedmann-Robertson-Walker (FRW) cosmology, we consider an exotic background fluid called modified generalized Chaplygin gas (MGCG) whose equation of state is as follows based on Ref.[19]

$$
p=-\frac{\alpha}{1+\alpha} \rho-\frac{1}{1+\alpha} \frac{\vartheta(z)}{\rho^{\alpha}}
$$

where $\alpha$ is a constant and $\vartheta(z)$ is a function of redshift $z$. Since the MGCG is another new unified model with two dark sectors (namely, at early times the energy density behaves as dark matter: $\rho \propto(1+z)^{3}$; while at late times it behaves like a quiessence dark energy: $\left.\rho \propto(1+z)^{3\left(1+\omega_{D E}\right)}\right)$, it could be supposed the energy density of the MGCG as follows

$$
\rho=\left[\kappa(1+z)^{3(1+\alpha)}+\lambda(1+z)^{3\left(1+\omega_{D E}\right)(1+\alpha)}\right]^{\frac{1}{1+\alpha}},
$$


where the parameter of state for dark energy $\omega_{D E}$ is a constant and should be taken as any value in the range $(-1.46,-0.78)\left[21\right.$. In Sec. 4 , we will further constrain the $\omega_{D E}$ in our model. Then the pressure of MGCG yields

$$
p=-\frac{\alpha\left[\kappa(1+z)^{3(1+\alpha)}+\lambda(1+z)^{3\left(1+\omega_{D E}\right)(1+\alpha)}\right]+\vartheta(z)}{(1+\alpha)\left[\kappa(1+z)^{3(1+\alpha)}+\lambda(1+z)^{3\left(1+\omega_{D E}\right)(1+\alpha)}\right]^{\alpha /(1+\alpha)}} .
$$

On the other hand, the whole pressure $p_{\text {total }}$ and energy density $\rho_{\text {total }}$ satisfy the conservation equation

$$
\dot{\rho}_{\text {total }}+3 H\left(\rho_{\text {total }}+p_{\text {total }}\right)=0 .
$$

According to conservation of baryons, the continuity equation is separated into two parts:

$$
\begin{aligned}
& \dot{\rho}_{B}+3 H\left(\rho_{B}+p_{B}\right)=0, \\
& \dot{\rho}+3 H(\rho+p)=0,
\end{aligned}
$$

where subscript "B" denotes baryons. Using the Eqs. (3), (44) and (7), we obtain

$$
\begin{aligned}
\vartheta(z) & =-\left\{\left[\omega_{D E}+\alpha\left(1+\omega_{D E}\right)\right] \lambda(1+z)^{3\left(1+\omega_{D E}\right)(1+\alpha)}\right. \\
& \left.+\alpha \kappa(1+z)^{3(1+\alpha)}\right\} .
\end{aligned}
$$

Furthermore, the pressure of dark energy $p_{D E}$, the energy density of dark energy $\rho_{D E}$ and the one of dark matter $\rho_{D M}$ can be respectively written as

$$
\begin{aligned}
p_{D E} & =p \\
& =\frac{\omega_{D E} \lambda(1+z)^{3\left(1+\omega_{D E}\right)(1+\alpha)}}{\left[\kappa(1+z)^{3(1+\alpha)}+\lambda(1+z)^{3\left(1+\omega_{D E}\right)(1+\alpha)}\right]^{\alpha /(1+\alpha)}}, \\
\rho_{D E} & =\frac{p_{D E}}{\omega_{D E}} \\
& =\frac{\lambda(1+z)^{3\left(1+\omega_{D E}\right)(1+\alpha)}}{\left[\kappa(1+z)^{3(1+\alpha)}+\lambda(1+z)^{3\left(1+\omega_{D E}\right)(1+\alpha)}\right]^{\alpha /(1+\alpha)}}, \\
\rho_{D M} & =\rho-\rho_{D E} \quad \kappa(1+z)^{3(1+\alpha)} \\
& =\frac{\left.{ }^{3\left(1+\omega_{D E}\right)(1+\alpha)}\right]^{\alpha /(1+\alpha)}}{\left[\kappa(1+z)^{3(1+\alpha)}+\lambda(1+z)^{3\left(1+\omega^{2}\right.}\right.} .
\end{aligned}
$$

From Eqs. (30), (10) and (11), it gives that

$$
\begin{aligned}
\kappa+\lambda & =\rho_{0}^{1+\alpha}=\left(\rho_{0 D M}+\rho_{0 D E}\right)^{1+\alpha}=\rho_{0 C}^{1+\alpha}\left[1-\Omega_{0 B}\right]^{1+\alpha}, \\
\rho_{0 D M} & =\frac{\kappa}{[\kappa+\lambda]^{\alpha /(1+\alpha)}}, \\
\rho_{0 D E} & =\frac{\lambda}{[\kappa+\lambda]^{\alpha /(1+\alpha)}},
\end{aligned}
$$


where $\rho_{0 C}, \rho_{0}, \rho_{0 D M}$ and $\rho_{0 D E}$ are the present values of $\rho_{\text {total }}, \rho, \rho_{D M}$ and $\rho_{D E}$, respectively and the label " 0 " denotes today's evaluated quantities. Parameters $\kappa$ and $\lambda$ can then be written as

$$
\kappa=\Omega_{0 D M} \rho_{0 C}^{1+\alpha}\left[1-\Omega_{0 B}\right]^{\alpha}, \quad \lambda=\Omega_{0 D E} \rho_{0 C}^{1+\alpha}\left[1-\Omega_{0 B}\right]^{\alpha} .
$$

Substituting Eq. (15) to Eq. (8), we have

$$
\begin{aligned}
\vartheta(z) & =-\rho_{0 C}^{1+\alpha}\left[1-\Omega_{0 B}\right]^{\alpha}(1+z)^{3(1+\alpha)}\left\{\alpha \Omega_{0 D M}\right. \\
& \left.+\Omega_{0 D E}\left[\omega_{D E}+\alpha\left(1+\omega_{D E}\right)\right](1+z)^{3 \omega_{D E}(1+\alpha)}\right\} .
\end{aligned}
$$

Then, the equation of state for the MGCG model reads as

$$
\chi(z) \equiv \frac{p}{\rho}=\frac{\omega_{D E} \Omega_{0 D E}(1+z)^{3\left(1+\omega_{D E}\right)(1+\alpha)}}{\Omega_{0 D M}(1+z)^{3(1+\alpha)}+\Omega_{0 D E}(1+z)^{3\left(1+\omega_{D E}\right)(1+\alpha)}} .
$$

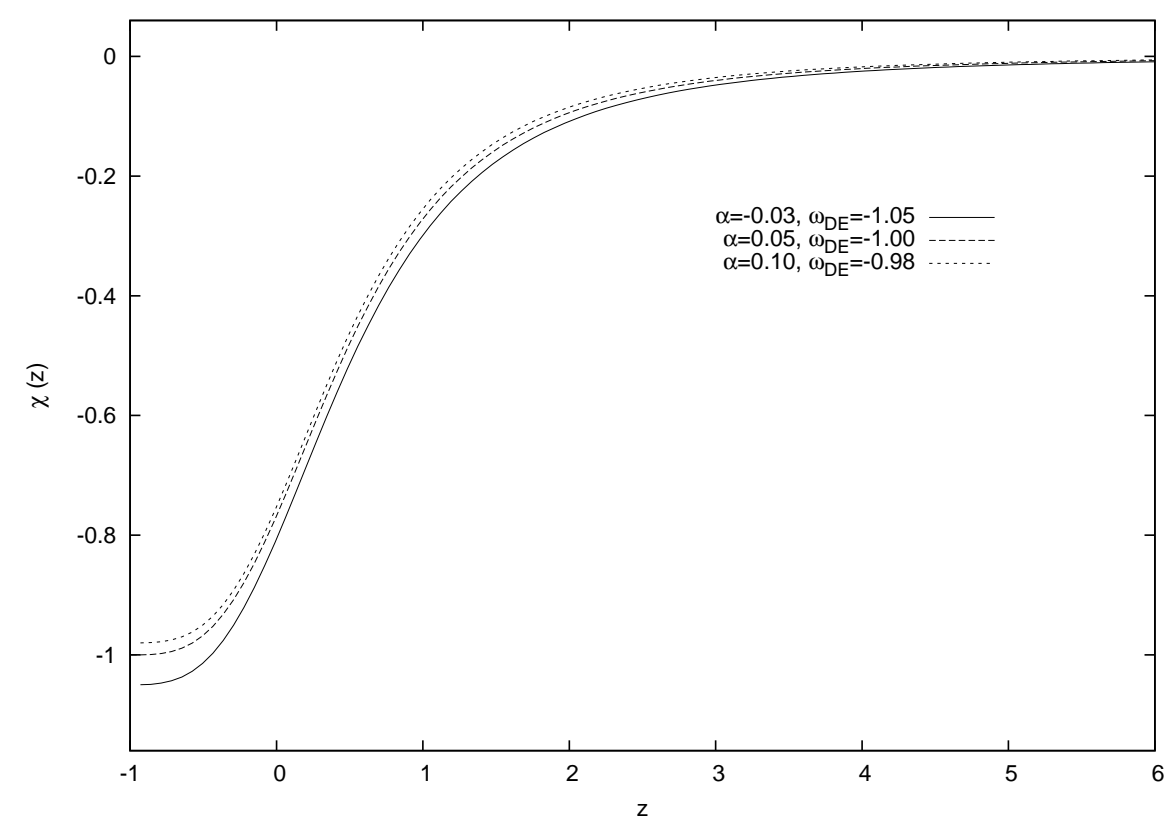

Figure 1. The equation of state for the MGCG $\chi(z)$ as the function of redshift. Where $\Omega_{0 B}=0.0449, \Omega_{0 D M}=0.222$ and $\Omega_{0 D E}=0.734$.

The values of $\Omega_{O D M}$ and $\Omega_{0 D E}$ depend on the model used in the data precessing. For example, both the Wilknson Microware Anisotropy Probe (WMAP) [24] and twodegree field Galaxy Redshift Survey (2dFGRS) [25] used $\Lambda$ CDM model of the universe. Besides, cosmological parameters are also constrained with other models. In this papar, the current density parameters used in the plots are $\Omega_{0 B}=0.0449 \pm 0.0028$, $\Omega_{0 D M}=0.222 \pm 0.026$ and $\Omega_{0 D E}=0.734 \pm 0.029$ based on WMAP7 data 24]. Later, we will give the constraints of $\alpha$ and $\omega_{D E}$ in section 4. From Eq.(17), we can plot the function $\chi(z)$ as the function of redshift $\mathrm{z}$ ( see Fig. 1). $\chi(z)$ is always negative from past to future. And we can see the effect of the parameter $\alpha$ and $\omega_{D E}$ on the $\chi(z)$. The evolution of $\chi(z)$ is mostly flat in the high redshift $z>1$ and is very steep as redshift $z$ becomes low. 


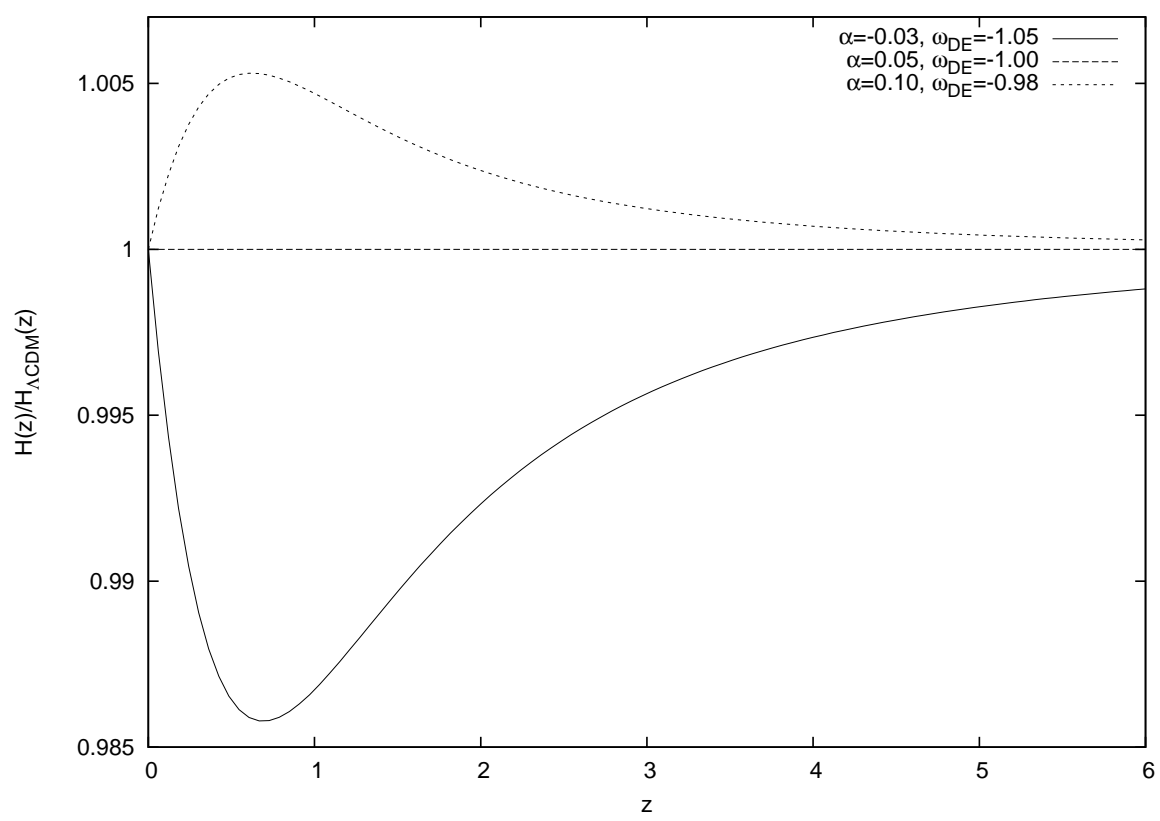

Figure 2. The Hubble parameter in units of $H_{\Lambda C D M}$ for the MGCG as the function of redshift. The priors $\Omega_{0 D M}=0.222, \Omega_{0 D E}=0.734$ and $\Omega_{0 B}=0.0449$ have been used.

When we consider a spatially flat FRW universe with the exotic background MGCG fluid and the baryon component, the Friedmann equation can be written as:

$$
H^{2}=\frac{8 \pi G}{3} \rho_{\text {total }}
$$

Then, we obtain

$$
\begin{aligned}
E^{2}(z) & \equiv \frac{H^{2}}{H_{0}^{2}} \\
& =\left(1-\Omega_{0 B}\right)^{\frac{\alpha}{1+\alpha}}\left[\Omega_{0 D M}(1+z)^{3(1+\alpha)}+\Omega_{0 D E}(1+z)^{3\left(1+\omega_{D E}\right)(1+\alpha)}\right]^{\frac{1}{1+\alpha}} \\
& +\Omega_{0 B}(1+z)^{3} .
\end{aligned}
$$

The evolution of the Hubble parameter in units of $H_{\Lambda \mathrm{CDM}}$ is plotted in Fig. 2, During the cosmological evolution, the behavior of $H$ is similar to $H_{\Lambda \mathrm{CDM}}$ at very early time, and when $z \rightarrow 0, H$ is approaching $\Lambda$ CDM.

By using the above equations, the densities of different components of the universe $\Omega_{D M}, \Omega_{D E}$ and $\Omega_{B}$ can be respectively derived as

$$
\begin{aligned}
& \Omega_{D M}=\frac{\left(1-\Omega_{0 B}\right)^{\frac{\alpha}{1+\alpha}} \Omega_{0 D M}(1+z)^{3}}{E^{2}(z)\left[\Omega_{0 D M}+\Omega_{0 D E}(1+z)^{3 \omega_{D E}(1+\alpha)}\right]^{\alpha /(1+\alpha)}}, \\
& \Omega_{D E}=\frac{\left(1-\Omega_{0 B}\right)^{\frac{\alpha}{1+\alpha}} \Omega_{0 D E}(1+z)^{3\left(1+\omega_{D E}\right)}}{E^{2}(z)\left[\Omega_{0 D M}(1+z)^{-3 \omega_{D E}(1+\alpha)}+\Omega_{0 D E}\right]^{\alpha /(1+\alpha)}}, \\
& \Omega_{B}=\frac{\Omega_{0 B}(1+z)^{3}}{E^{2}(z)} .
\end{aligned}
$$




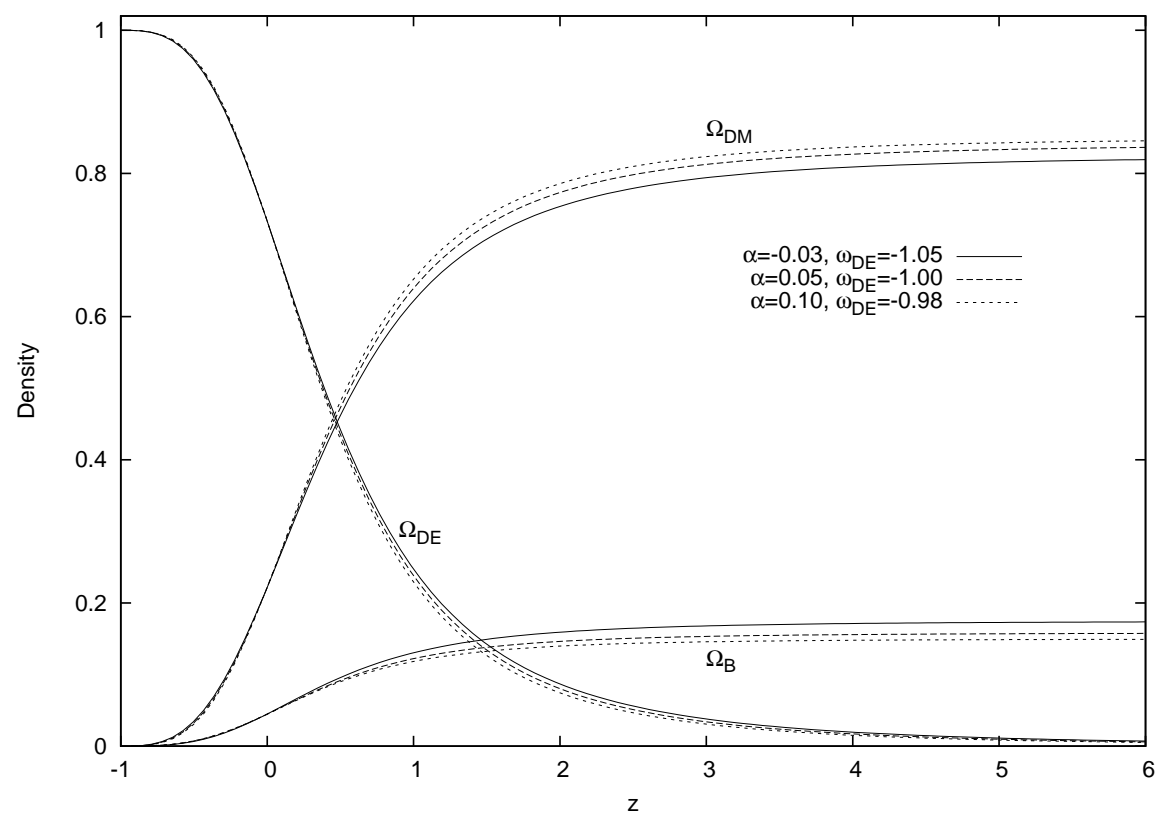

Figure 3. The densities of $\Omega_{D E}, \Omega_{D M}$ and $\Omega_{B}$ as the functions of redshift.

The relationships between $\Omega_{i}$ ( $i$ respectively denotes baryons, dark matter and dark energy) and redshift $z$ are shown in Fig. 3 with the prior of $\Omega_{0 D M}=0.222, \Omega_{0 D E}=0.734$ and $\Omega_{0 B}=0.0449$. Based on Fig. 3, the evolutions of baryons, dark matter and dark energy under MGCG model are consistent with what are recognized [21, 22]. An very important redshift described the epoch when the densities in dark matter and dark energy are equal is $\left(1+z_{e q}\right)^{-3 \omega_{D E}(1+\alpha)}=\Omega_{0 D E} / \Omega_{0 D M}$. And $z_{e q} \simeq 0.48$ for $\alpha=-0.03, \omega_{D E}=-1.05, z_{e q} \simeq 0.46$ for $\alpha=0.05, \omega_{D E}=-1.00, z_{e q} \simeq 0.45$ for $\alpha=0.10, \omega_{D E}=-0.98$ respectively. From Fig. 3 we also know that the dominance of the dark energy leads to the acceleration expansion of our universe. The increasing density of dark energy is the reason why expansion of our universe transited from deceleration to acceleration. This transition will be described by our model in more detail with the deceleration parameter $q$.

The deceleration parameter is

$$
\begin{aligned}
q & \equiv-\frac{\ddot{a}}{a H^{2}} \\
& =-1-\frac{\dot{H}}{H^{2}} \\
& =-1+\frac{3(1+z)^{3}}{2 E^{2}(z)}\left(1-\Omega_{0 B}\right)^{\alpha /(1+\alpha)}\left\{\left[\Omega_{0 D M}+\Omega_{0 D E}(1+z)^{3 \omega_{D E}(1+\alpha)}\right]^{-\frac{\alpha}{1+\alpha}}\right. \\
& \left.\times\left[\Omega_{0 D M}+\left(1+\omega_{D E}\right) \Omega_{0 D E}(1+z)^{3 \omega_{D E}(1+\alpha)}\right]+\Omega_{0 B}\right\}
\end{aligned}
$$

Thus we can give the following results: the present deceleration parameter $q_{0} \approx-0.65$ according to $z=0$ and the transition redshift $z_{T} \approx 0.70$ according to $q=0$ when taking $\alpha=-0.03, \omega_{D E}=-1.05, q_{0} \approx-0.60$ and $z_{T} \approx 0.65$ for $\alpha=0.05, \omega_{D E}=-1.00$, and $q_{0} \approx-0.58$ and $z_{T} \approx 0.60$ for $\alpha=0.10, \omega_{D E}=-0.98$, which are consistent with 
observations. The deceleration parameter as the function of redshift is shown in Fig. 4 . According to Fig. 4, the expansion of the universe is from slowing down $(q>0)$ in the past to speeding up $(q<0)$ at present time and in the future. And when $q>0$ is at the high redshift, $q$ changes very slow with redshift $z$; when $q<0$ is at the recent time, and $q$ changes faster and faster with redshift $z$.

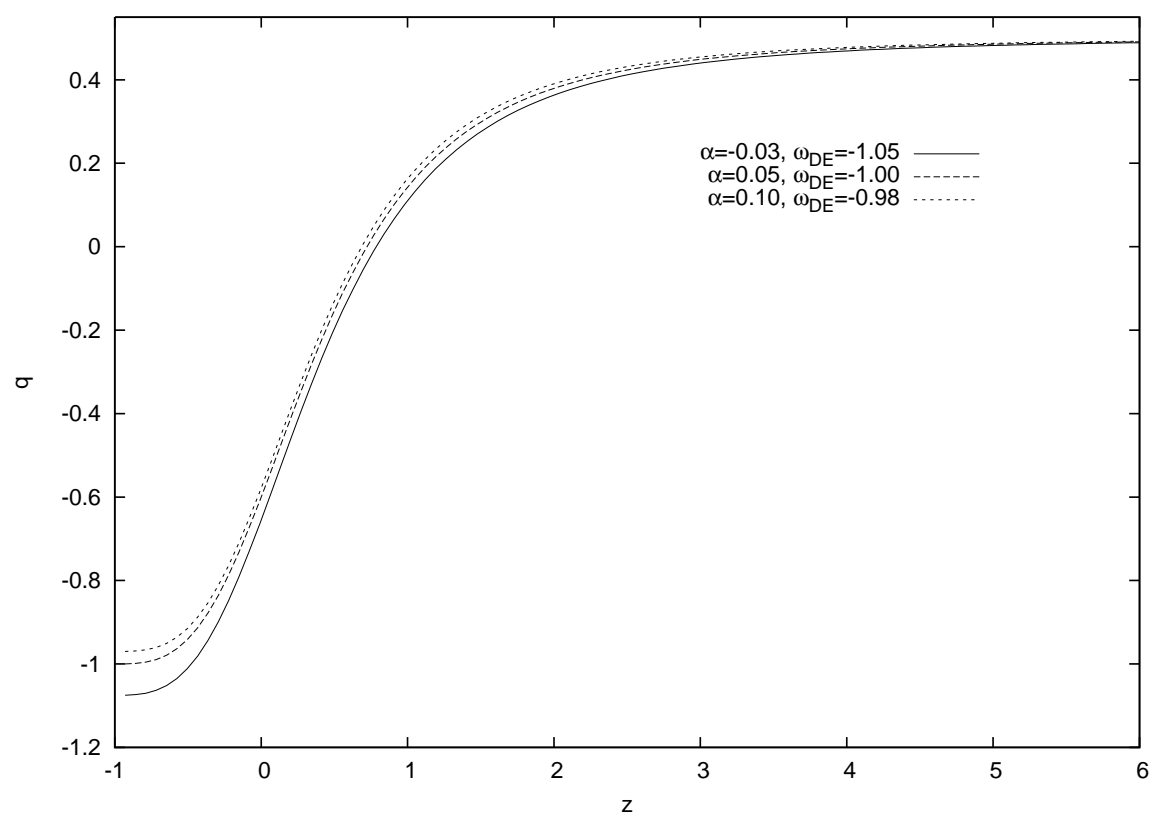

Figure 4. The deceleration parameter $q$ as the function of redshift. The priors $\Omega_{0 D M}=0.222, \Omega_{0 D E}=0.734$ and $\Omega_{0 B}=0.0449$ have been used.

Now, we compare some cosmological characters between CG, GCG, NGCG, MCG and MGCG (see Table 1). In order to distinguish these model, we use the same symbols as above. From Table 1, we can see the EOSs of these models so that they have some different characters. First of all, CG and GCG can be treated as a unified fluid whose behaves like pressureless dust at early times and like a cosmological constant during very late times. For NGCG, it is considered as $X$-CDM, where $X$ means quiessence, since the authors in Ref. [20] extended $A$ of GCG model. And MCG is the mixture of a barotropic perfect fluid and a generalized Chaplygin gas. At the same time, considering $A$ of Eq. (1) changed with redshift $z$, we proposed MGCG.

Among them, GCG is a extended model of CG. When $\alpha=1$, GCG model becomes CG. And NGCG is a extended model of GCG. When $\tilde{A}(z)=$ const., NGCG reduces to GCG. MGCG is a extended version of MCG. When $\vartheta(z)=$ const., MGCG yields MCG. Meanwhile, both GCG and MCG can be conceived as ETF, in which GCG corresponds to the simplest model driven by a constant potential[19]. It is worth of noted that $\mathrm{CG}$, GCG, NGCG, MCG and MGCG are all interact models. Namely, there are energy exchange between dark matter and dark energy. Basically, the behaviour of the energy exchange can be presented as $\rho_{D M} / \rho_{D E}$. We can see the transfer direction of the energy flow for $\mathrm{CG}$ is from dark matter to dark energy. However, the transfer direction of the 
Table 1. Compare CG, GCG, NGCG, MCG and MGCG

\begin{tabular}{lllll}
\hline Models & EOS & A & Parameter $(\mathrm{s})$ & $\rho_{D M} / \rho_{D E}$ \\
\hline CG & $p=-\frac{A}{\rho}$ & Const. & $A$ & $\sim(1+z)^{3}$ \\
GCG & $p=-\frac{A}{\rho^{\alpha}}$ & Const. & $A$ and $\alpha$ & $\sim(1+z)^{3(1+\alpha)}$ \\
NGCG & $p=-\frac{\tilde{A}(z)}{\rho^{\alpha}}$ & Function of $z$ & $\omega_{D E}$ and $\alpha$ & $\sim(1+z)^{-3 \omega_{D E}(1+\alpha)}$ \\
MCG & $p=-\frac{\alpha}{1+\alpha} \rho-\frac{1}{1+\alpha} \frac{A}{\rho^{\alpha}}$ & Const. & $A$ and $\alpha$ & $\sim(1+z)^{3(1+\alpha)}$ \\
MGCG & $p=-\frac{\alpha}{1+\alpha} \rho-\frac{1}{1+\alpha} \frac{\vartheta(z)}{\rho^{\alpha}}$ & Function of $z$ & $\omega_{D E}$ and $\alpha$ & $\sim(1+z)^{-3 \omega_{D E}(1+\alpha)}$ \\
\hline
\end{tabular}

energy flow for the others only depend on the parameter $\alpha$ if $\alpha \neq 0$. When $\alpha>0$, the transfer direction of the energy flow is from dark matter to dark energy. When $\alpha<0$, the direction of transfer is inverse. Thus, constraints of parameters such as $\alpha$ and $\omega_{D E}$ from observational data and theoretical analysis will play an important role in understanding the physical nature of these models. For GCG, authors in Ref.[27] obtain $\alpha<10^{-5}$ or $\alpha \geq 3$ (the sound speed is larger than the speed of light) by using gauge-invariant analysis of perturbation. For NGCG, constraints of parameters are $1+\alpha=1.06_{-0.16}^{+0.20}$ and $\omega_{D E}=-0.98_{-0.20}^{+0.15}$ by observational constraints from SNe Ia, CMB and LSS data [20]. In section 4 , we will constrain $\alpha$ and $\omega_{D E}$ in our model by the maximum likelihood estimation.

\section{Statefinder diagnostic}

Dark energy models such as the cosmological constant [4, 5], quintessence 6], Kessence [7], Chaplygin gas[13] and quintom[10] etc. have properties which can be model-dependent. In order to distinguish the very distinct and competing cosmological scenarios involving dark energy, a sensitive and robust diagnostic of dark energy is needed. So a new diagnostic of dark energy called "Statefinder" diagnostic has been constructed by Sahni et al.23] who were using both the second and the third derivatives of the scale factor $a$.

The statefinder pair $\{r, s\}$, in addition to the oldest and most well-known geometric variables $H$ and $q$, defines two new cosmological parameters

$$
\begin{aligned}
& r \equiv \frac{\dddot{a}}{a H^{3}}, \\
& s \equiv \frac{r-1}{3(q-1 / 2)} .
\end{aligned}
$$

An important property of the Statefinder is that spatially flat $\Lambda \mathrm{CDM}$ corresponds to the fixed point

$$
\left.\{r, s\}\right|_{\Lambda \mathrm{CDM}}=\{1,0\} .
$$

Clearly an important requirement of any diagnostic is that it permits us to tell difference between a given dark energy model and the simplest of all models - the cosmological 
constant just as demonstrated in[23]-[28]. By using the $r(s)$ evolution diagram, the discrimination between a given dark energy model and the $\Lambda$ CDM scenario can be clearly identified. And it is more worth of noted that the Statefinder diagnostic combined with future SNAP 29] observations may possibly be used to distinguish between different dark energy models.

Based on the Eqs. (24) and (25), we obtain the Statefinder parameters for the MGCG as follows

$$
\begin{aligned}
& r=1+3 \frac{\dot{H}}{H^{2}}+\frac{\ddot{H}}{H^{3}} \equiv 1+3 \Upsilon(z)+\Theta(z), \\
& s=-\frac{6 \dot{H} / H^{2}+2 \ddot{H} / H^{3}}{9+6 \dot{H} / H^{2}} \equiv-\frac{6 \Upsilon(z)+2 \Theta(z)}{9+6 \Upsilon(z)},
\end{aligned}
$$

where

$$
\begin{aligned}
\Upsilon(z) & =\frac{\dot{H}}{H^{2}} \\
& =-\frac{3}{2}-\frac{3}{2} \omega_{D E} \Omega_{0 D E}(1+z)^{3 \omega_{D E}(1+\alpha)}\left(1-\Omega_{0 B}\right)^{\alpha /(1+\alpha)}\left\{\left(1-\Omega_{0 B}\right)^{\alpha /(1+\alpha)}\right. \\
& \times\left[\Omega_{0 D M}+\Omega_{0 D E}(1+z)^{3 \omega_{D E}(1+\alpha)}\right]+\Omega_{0 B}\left[\Omega_{0 D M}\right. \\
& \left.\left.+\Omega_{0 D E}(1+z)^{3 \omega_{D E}(1+\alpha)}\right]^{\alpha /(1+\alpha)}\right\}^{-1}
\end{aligned}
$$

and

$$
\begin{aligned}
\Theta(z) & =\frac{\ddot{H}}{H^{3}} \\
& =\frac{9}{2}+\frac{9}{2}\left(1-\Omega_{0 B}\right)^{\alpha /(1+\alpha)}\left\{2 \omega_{D E} \Omega_{0 D E}(1+z)^{3 \omega_{D E}(1+\alpha)}\right. \\
& +(1+\alpha) \omega_{D E}^{2} \Omega_{0 D E}(1+z)^{3 \omega_{D E}(1+\alpha)} \\
& -\alpha \omega_{D E}^{2} \Omega_{0 D E}^{2}(1+z)^{6 \omega_{D E}(1+\alpha)}\left[\Omega_{0 D M}\right. \\
& \left.\left.+\Omega_{0 D E}(1+z)^{3 \omega_{D E}(1+\alpha)}\right]^{-1}\right\}\left\{\left(1-\Omega_{0 B}\right)^{\alpha /(1+\alpha)}\right. \\
& \times\left[\Omega_{0 D M}+\Omega_{0 D E}(1+z)^{3 \omega_{D E}(1+\alpha)}\right] \\
& \left.+\Omega_{0 B}\left[\Omega_{0 D M}+\Omega_{0 D E}(1+z)^{3 \omega_{D E}(1+\alpha)}\right]^{\alpha /(1+\alpha)}\right\}^{-1}
\end{aligned}
$$

Then, the evolution trajectories of our model in the plane of the Statefinder parameters can also be plotted (see Fig. 5 and Fig. [6).

Since different dark energy models have distinct evolution trajectories in the plane of the Statefinder parameters, we can distinguish between various dark energy models and the MGCG. Just as the literatures[23, 28] show: the $\Lambda$ CDM scenario corresponds to the fixed point $\{r=1, s=0\}$; the standard cold dark matter (SCDM) scenario corresponds to the point $\{r=1, s=1\}$; the Statefinder parameters plane of quiessence are some vertical segments where $r$ decreases monotonically from 1 to $1+\frac{9}{2} \omega_{D E}\left(1+\omega_{D E}\right)$ and $s$ 


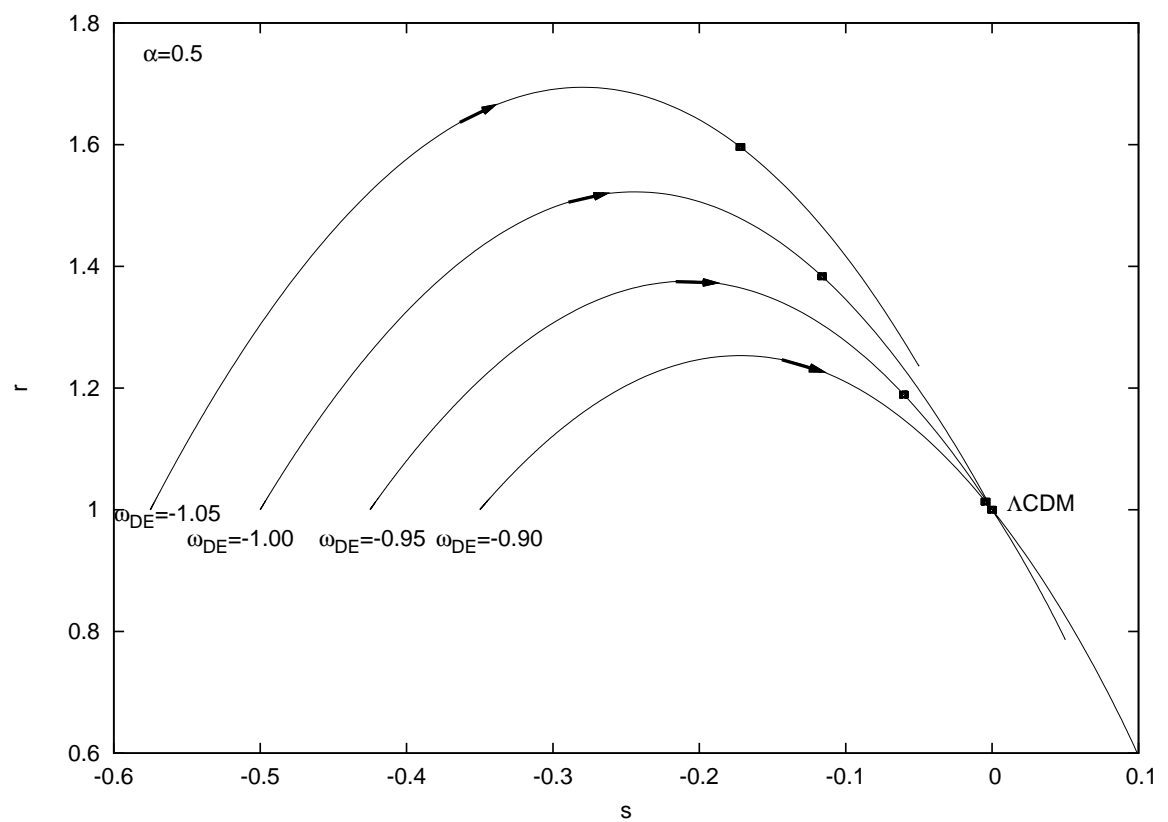

Figure 5. The Statefinder $r(s)$ evolution diagram when we fix $\alpha$ and vary $\omega_{D E}$. Dots locate today's values and arrows denote the direction of evolution for the MGCG.

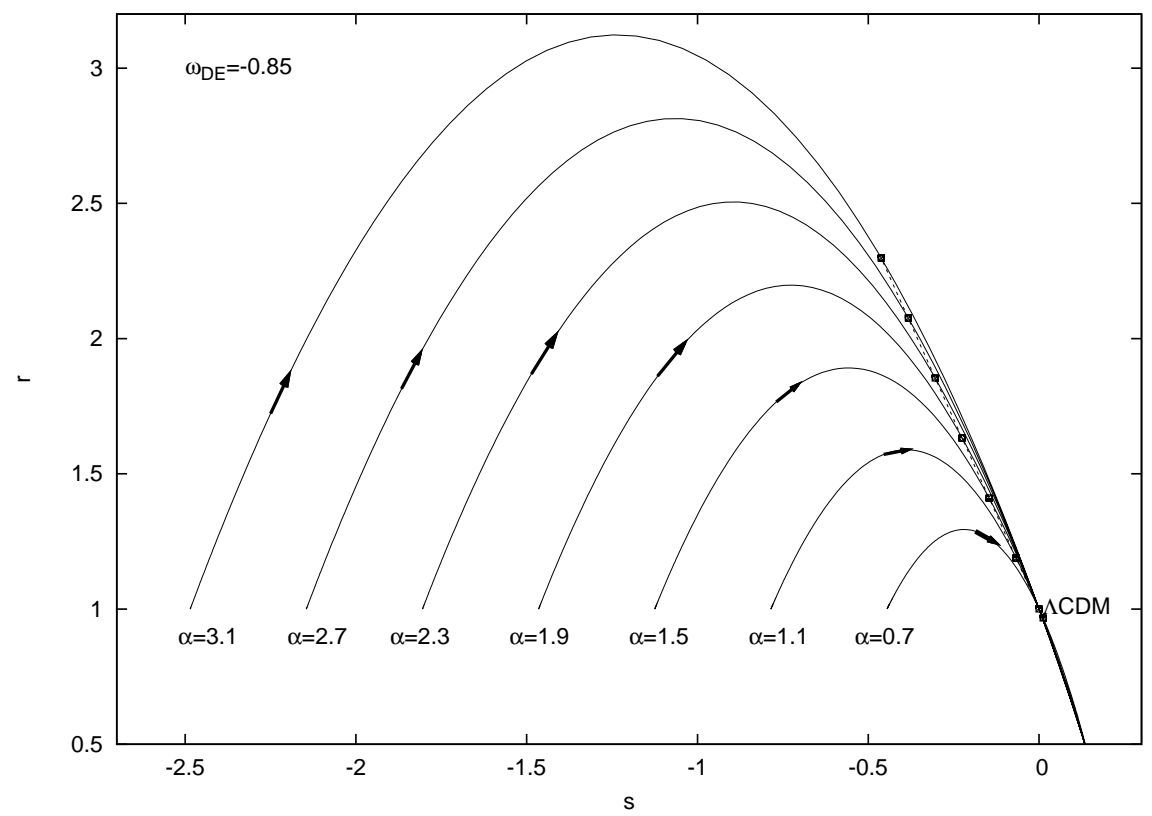

Figure 6. The Statefinder $r(s)$ evolution diagram when we fix $\omega_{D E}$ and vary $\alpha$. Dots locate today's values and arrows denote the direction of evolution for the MGCG. 
remains constant at $1+\omega_{D E}$; the quintessence tracker models have typical trajectories similar to arcs of an upward parabola lying in the regions $\{r<1, s>0\}$.

Now, we can see the Statefinder parameters plane of the MGCG from Fig. 5 and Fig. 6. In Fig. 5, we fix $\alpha=0.5$ and vary $\omega_{D E}$ as $-1.05,-1.00,-0.95$ and -0.90 respectively. Where $\omega_{D E}=-1.00$ exhibits a complete downward parabola, while the others correspond to some broken parabolas. And from this diagram, we can see the Statefinder trajectory begins with $r=1$. Besides, the cases of $\omega_{D E}<-1$ never arrive at the fixed point $\{1,0\}$ and when $\omega_{D E}>-1$ the trajectories must pass through this point. Finally, we can see that the less $\omega_{D E}$, the smaller today's value $s_{0}$ and the larger today's value $r_{0}$. However, in Fig. 6, we fix $\omega_{D E}=-0.85$ and vary $\alpha$ as $3.1,2.7,2.3,1.9,1.51$, 1.1 and 0.7 respectively. It is interesting to see that the trajectories can pass through the fixed point $\{1,0\}$. And when fixed $\omega_{D E}$, the less $\alpha$, the smaller today's value $s_{0}$ and the larger today's value $r_{0}$. We notice the present Statefinder points as well as the fixed

point $\{1,0\}$ locate on a straight line which means the relationship between $r_{0}$ and $s_{0}$ is linear when we fix $\omega_{D E}$. That is because the following relationships

$$
\begin{aligned}
& r_{0}=1+\frac{9}{2} \omega_{D E} \Omega_{0 D E}\left[1+(1+\alpha) \omega_{D E}-\frac{\alpha \omega_{D E} \Omega_{0 D E}}{1-\Omega_{0 B}}\right], \\
& s_{0}=1+(1+\alpha) \omega_{D E}-\frac{\alpha \omega_{D E} \Omega_{0 D E}}{1-\Omega_{0 B}},
\end{aligned}
$$

so that

$$
r_{0}=1+\frac{9}{2} \omega_{D E} \Omega_{0 D E} s_{0}
$$

Through above, the separation between distinct families of dark energy models is very remarkable when we analyze evolutionary trajectories using the Statefinder pair $\{r, s\}$. Forthcoming space-based missions such as SNAP are expected to greatly increase and improve the current Type Ia supernovae inventory, and maybe can identify which kind of dark energy model.

\section{Constraints on MGCG}

\subsection{The sound speed}

Since our model can be considered as a new scenario for unified dark matter and dark energy, we have to study its density perturbations and the structure formation, we also have to investigate its adiabatic sound speed. So, in this section, we consider some effective parameters for the MGCG such as $\alpha$ and $\omega_{D E}$ by the maximum likelihood estimation. If the MGCG is considered as a perfect fluid satisfying equation (2), then the MGCG component will cluster gravitationally with the adiabatic sound speed given by

$$
c_{s}^{2} \equiv \frac{\partial p}{\partial \rho}
$$




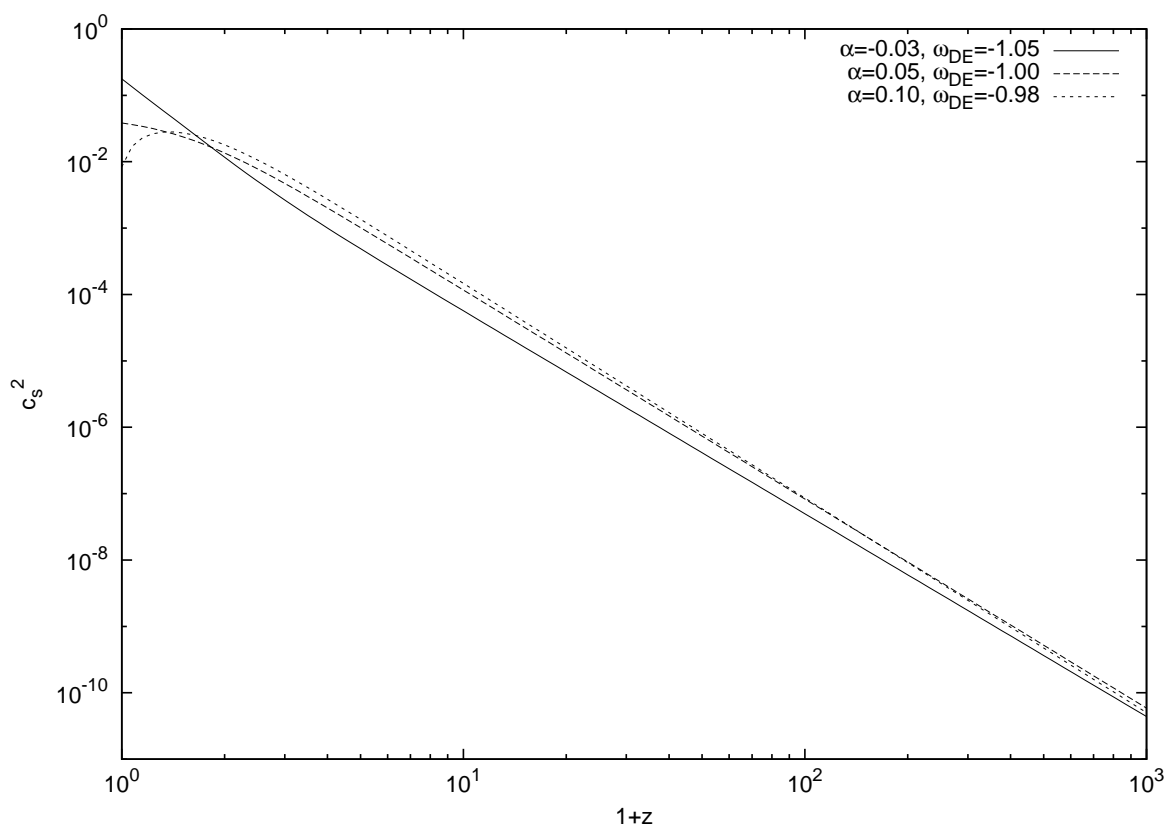

Figure 7. The evolution of the sound speed squared of the MGCG as a function of redshift for $\alpha=-0.03,0.05$ and 0.10 .

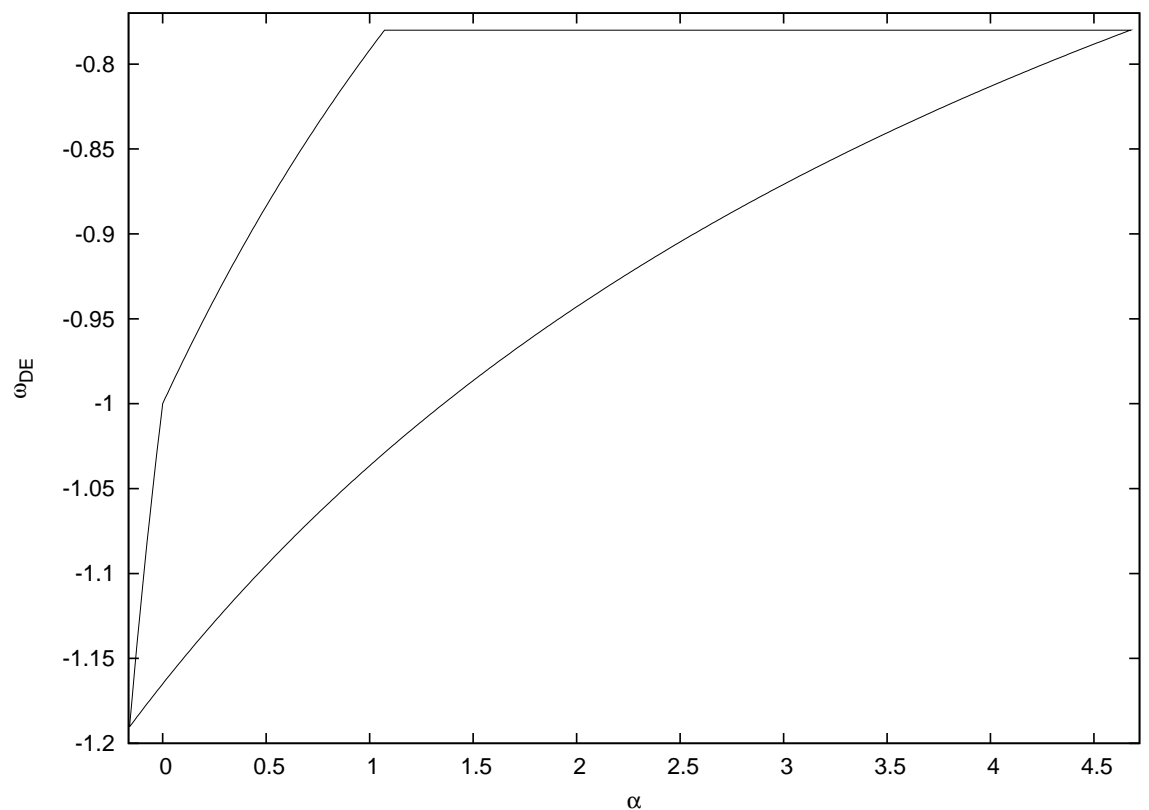

Figure 8. The region inside the curve is the domain of parameters $\alpha$ and $\omega_{D E}$ which is constraints from the sound speed. 


$$
\begin{aligned}
& =-\frac{\alpha}{1+\alpha}-\frac{\alpha}{1+\alpha} \frac{\Omega_{0 D E}\left[\omega_{D E}+\alpha\left(1+\omega_{D E}\right)\right](1+z)^{3 \omega_{D E}(1+\alpha)}+\alpha \Omega_{0 D M}}{\Omega_{0 D E}(1+z)^{3 \omega_{D E}(1+\alpha)}+\Omega_{0 D M}} \\
& +\frac{\left(1+\omega_{D E}\right) \Omega_{0 D E}\left[\omega_{D E}+\alpha\left(1+\omega_{D E}\right)\right](1+z)^{3 \omega_{D E}(1+\alpha)}+\alpha \Omega_{0 D M}}{\left(1+\omega_{D E}\right) \Omega_{0 D E}(1+z)^{3 \omega_{D E}(1+\alpha)}+\Omega_{0 D M}} .
\end{aligned}
$$

From the above equation, we obtain $\alpha \neq-1$. However, when $\alpha<-1$, we can derive $\left.c_{s}^{2}\right|_{z \rightarrow \infty} \simeq \omega_{D E}<0$, and when $c_{s}^{2}$ is negative, the MGCG fluid is unstable. When $\alpha>-1$, we have $\left.c_{s}^{2}\right|_{z \rightarrow \infty} \simeq 0$. Thus, the parameter $\alpha$ must be greater than -1 because the limit of the sound speed was very small at early time and can not be larger than 1 even though in the future if our model can be as the unified dark matter-dark energy. From Fig. 7, we can see the slope of the curves for $c_{s}^{2}$ depends on $\alpha$ and $\omega_{D E}$. Besides, we clearly see that deep in the matter era the behavior of the MGCG closely resembles that of CDM.

To constrain $\alpha$ and $\omega_{D E}$ further, combined with the data of SNe Ia, CMB and 2dFGRS, it imposes a range on $\omega_{D E}:-1.46<\omega_{D E}<-0.78$ [21]. Through the limit of the sound speed $0<c_{s}^{2}<1$, when we use the Eq. (33), we can find the domain of $\alpha$ $\omega_{D E}$ plane (see Fig. 8). Then, we derive $-0.3<\alpha<4.7$ and $-1.19<\omega_{D E}<-0.78$. From Fig. 8, we can see the boundary of domain is constraints from the sound speed.

\subsection{The age of the universe}

By using a distance-independent method, Jimenez et al.[32] determined the age of globular clusters in the Milky Way as $t_{0}=13.5 \pm 2 \mathrm{Gyr}$. By using the white dwarfs cooling sequence method, Richer et al.33] and Hansen et al. [34] constrained the age of the globular cluster M4 to be $t_{0}=12.7 \pm 0.7 \mathrm{Gyr}$. Then, the age of the universe needs to satisfy the lower bound: $t_{0}>11-12 \mathrm{Gyr}$. Assuming $\Lambda \mathrm{CDM}$, WMAP7 data give the age of the universe $t_{0}=13.73 \pm 0.13 \mathrm{Gyr}[24$. Here we adopt model-independent astronomical observations of globular clusters as a criterion. Making use of the definition for the Hubble parameter $H=\dot{a} / a$ and the relationship between the scale factor and the redshift $1+z=1 / a$, then we have

$$
d t=\frac{d a}{a H}=-\frac{1}{(1+z) H} d z .
$$

Integrated the above equation, we obtain

$$
\begin{aligned}
t_{0} & =\int_{0}^{t_{0}} d t=\int_{0}^{\infty} \frac{d z}{H(1+z)} \\
& =\frac{1}{H_{0}} \int_{0}^{\infty} \frac{d z}{(1+z)^{5 / 2} \sqrt{\left(1-\Omega_{0 B}\right)^{\frac{\alpha}{1+\alpha}}\left[\Omega_{0 D M}+\Omega_{0 D E}(1+z)^{3 \omega_{D E}(1+\alpha)}\right]^{\frac{1}{1+\alpha}}+\Omega_{0 B}}}
\end{aligned}
$$

In Fig. 9, with using the age data of the universe, we plot the isochrones in $\alpha-\omega_{D E}$ plane under the boundary of domain constrained by $c_{s}^{2}$. Then, we can see that the parameters $\alpha$ and $\omega_{D E}$ are constrained by the age of the universe further. Since the luminosity distance for all models is given by the simple expression for a spatially flat universe

$$
H(z)=\left[\frac{d}{d z}\left(\frac{D_{L}(z)}{1+z}\right)\right]^{-1},
$$


using the Eqs. (35) $-(\underline{36})$, we have

$$
t_{0}=\left.\frac{D_{L}}{(1+z)^{2}}\right|_{0} ^{\infty}+\int_{0}^{\infty} \frac{D_{L}}{(1+z)^{3}} d z .
$$

From above equation, we can see that the luminosity distance is directly related to the age of the universe.

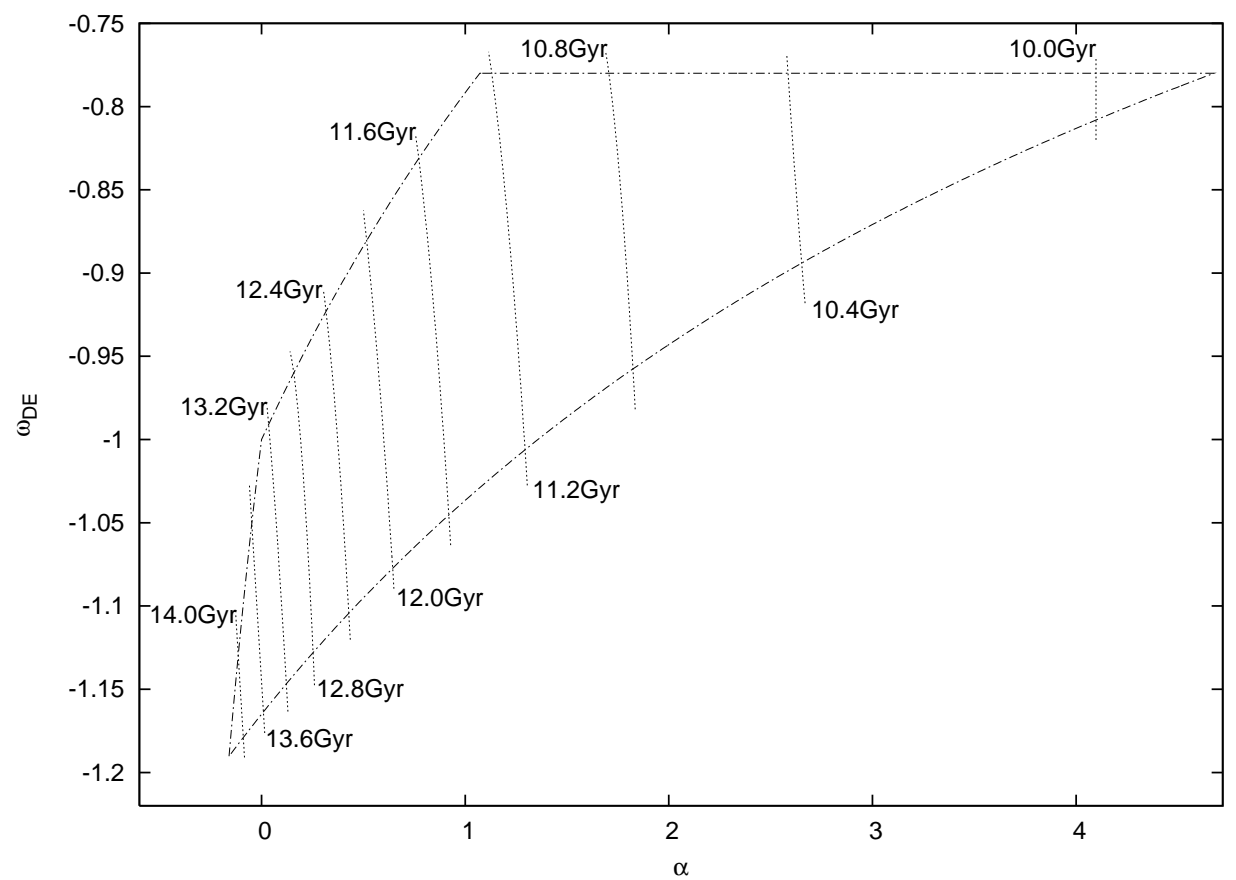

Figure 9. The dashed-dot curve is the domain of parameters $\alpha$ and $\omega_{D E}$ which is constraints from the sound speed. The dot lines is the isochrones for parameter $t_{0}$ in the $\alpha-\omega_{D E}$ plane.

\subsection{The linear density perturbations}

In this subsection, we will study the growth of density perturbations for the MGCG fluid in the linear regime on subhorizon scales. We will follow the analysis method in Ref. [13]. Firstly, we will introduce some quantities used in this part. The relationship between the comoving coordinate $(\vec{x}, t)$ and the physical coordinate $(\vec{r}, t)$ is $\vec{x}=\vec{r} / a$. The velocity in physical coordinate is $\vec{u}=\dot{\vec{r}}$. Furthermore, we can expand $\vec{u}=\dot{a} \vec{x}+\vec{v}$ where $\vec{v}$ is the first-order perturbation to the Hubble flow $\dot{a} \vec{x} . \rho_{D M}(\vec{x}, t)=\rho_{0 D M}(t)\left(1+\delta_{D M}(\vec{x}, t)\right)$ where $\rho_{0 D M}(t)$ is the background density and $\delta_{D M}(\vec{x}, t)$ is the first-order perturbation of $\rho_{0 D M} \cdot \phi(\vec{x}, t)$ is the first-order perturbation of Newtonian potential $\Phi$, which satisfies Poisson's equation $\nabla^{2} \Phi=4 \pi G a^{2} \rho_{D M}$ in the comoving coordinate.

Before discussing the linear density perturbations, we will research an explicit interaction between dark matter and dark energy in our model and depict this interaction through an energy exchange term $\Gamma$ which will be used in the linear density perturbed 
equations. From Eqs. (10)-(11), we can obtain the scaling behavior of the energy densities as follows

$$
\frac{\rho_{D M}}{\rho_{D E}}=\frac{\kappa}{\lambda}(1+z)^{-3 \omega_{D E}(1+\alpha)}=\frac{\Omega_{0 D M}}{\Omega_{0 D E}}(1+z)^{-3 \omega_{D E}(1+\alpha)},
$$

where there is an explicit interaction between dark matter and dark energy. This can be seen from the energy conservation equation more clearly, which can be written as

$$
\dot{\rho}_{D M}+3 H \rho_{D M}=-\left[\dot{\rho}_{D E}+3 H\left(\rho_{D E}+p_{D E}\right)\right] .
$$

Furthermore,

$$
\begin{aligned}
& \dot{\rho}_{D M}+3 H \rho_{D M}=\Gamma, \\
& \dot{\rho}_{D E}+3 H\left(\rho_{D E}+p_{D E}\right)=-\Gamma,
\end{aligned}
$$

where $\Gamma$ is the source term in the continuity equation due to the energy transferring between dark matter and quiessence dark energy in our model. From the above equations, we can respectively derive the effective equations of state for dark matter $\omega_{D M}^{e f f}$ and dark energy $\omega_{D E}^{e f f}$ in the MGCG scenario

$$
\begin{aligned}
\omega_{D M}^{e f f} & =-\frac{\Gamma}{3 H \rho_{D M}} \\
& =-\frac{\alpha \omega_{D E} \Omega_{0 D E}(1+z)^{3 \omega_{D E}(1+\alpha)}}{\Omega_{0 D M}+\Omega_{0 D E}(1+z)^{3 \omega_{D E}(1+\alpha)}}, \\
\omega_{D E}^{e f f} & =\omega_{D E}+\frac{\Gamma}{3 H \rho_{D E}} \\
& =\omega_{D E}(1+\alpha)-\frac{\alpha \omega_{D E} \Omega_{0 D E}(1+z)^{3 \omega_{D E}(1+\alpha)}}{\Omega_{0 D M}+\Omega_{0 D E}(1+z)^{3 \omega_{D E}(1+\alpha)}} .
\end{aligned}
$$

Now, we focus on the mass density perturbations in the linear approximation. In this paper, we apply the comoving coordinate $(\vec{x}, t)$ for a pressureless fluid with background density $\rho_{0 D M}$ and density contrast $\delta_{D M}$, with a source term $\Gamma$ due to the energy transfer from dark matter to quiessence dark energy. Based on Ref. [30], in the first-order approximation, the perturbed equations are written in the comoving coordinate as follows

$$
\begin{aligned}
& \frac{\partial \vec{v}}{\partial t}+\frac{\dot{a}}{a} \vec{v}=-\frac{\nabla \phi}{a}, \\
& \nabla \cdot \vec{v}=-a \frac{\partial \delta_{D M}}{\partial t}-a \Gamma \frac{\delta_{D M}}{\rho_{0 D M}}, \\
& \frac{1}{a^{2}} \nabla^{2} \phi=4 \pi G \rho_{0 D M} \delta_{D M},
\end{aligned}
$$

where $\delta_{D M}, \vec{v}$ and $\phi$ are respectively the first-order perturbations of $\rho_{0 D M}, \dot{a} \vec{x}$ and the Newtonian potential $\Phi$. Since the matter is so cold that the term proportional to the speed of sound $c_{s}^{2}$ in Eq. (44) can be omitted. It leads to disappearance of the term $\frac{c_{s}^{2}}{a} \nabla \delta_{D M}$ in the Eq. (44) [31]. Assuming that both the density contrast $\delta_{D M}$ and peculiar 


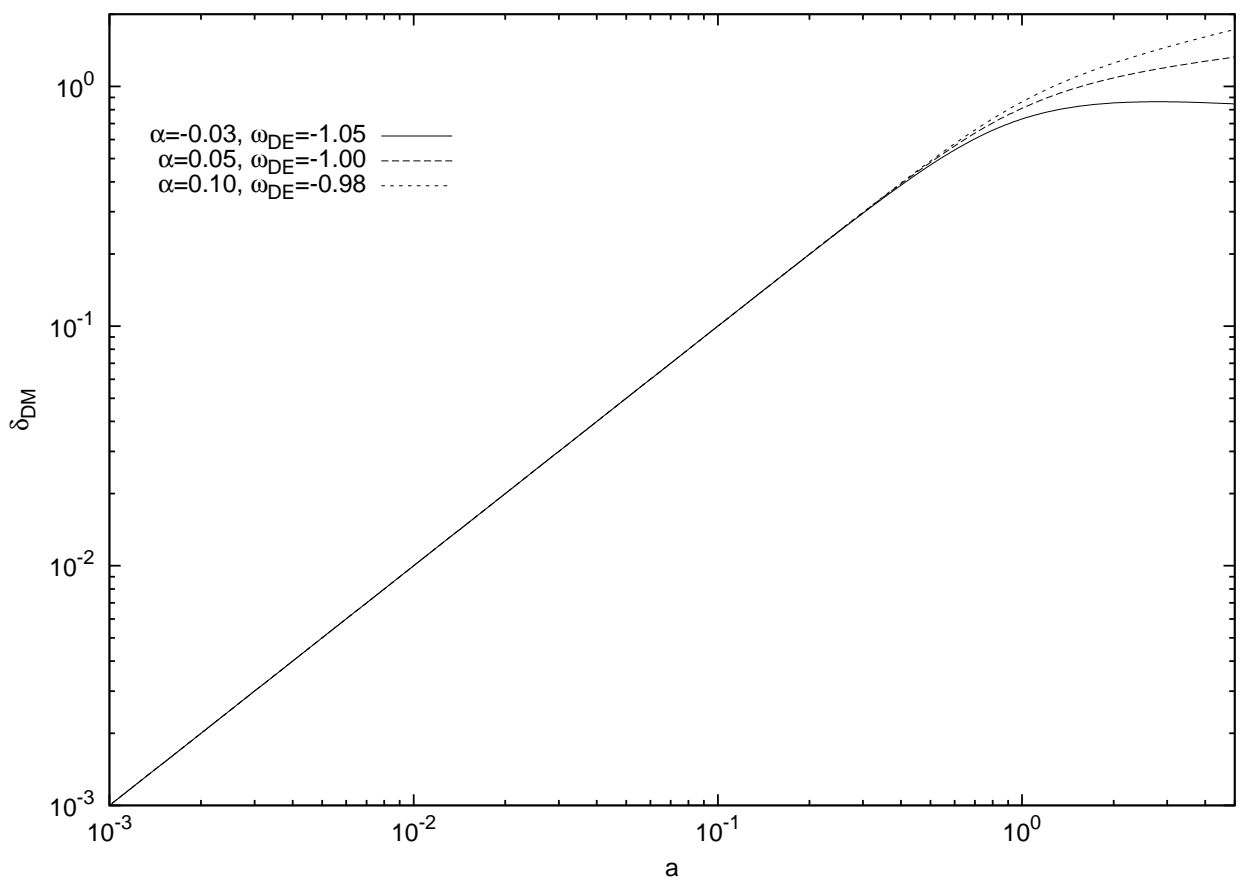

Figure 10. $\delta_{D M}$ as the function of scale factor $a$.

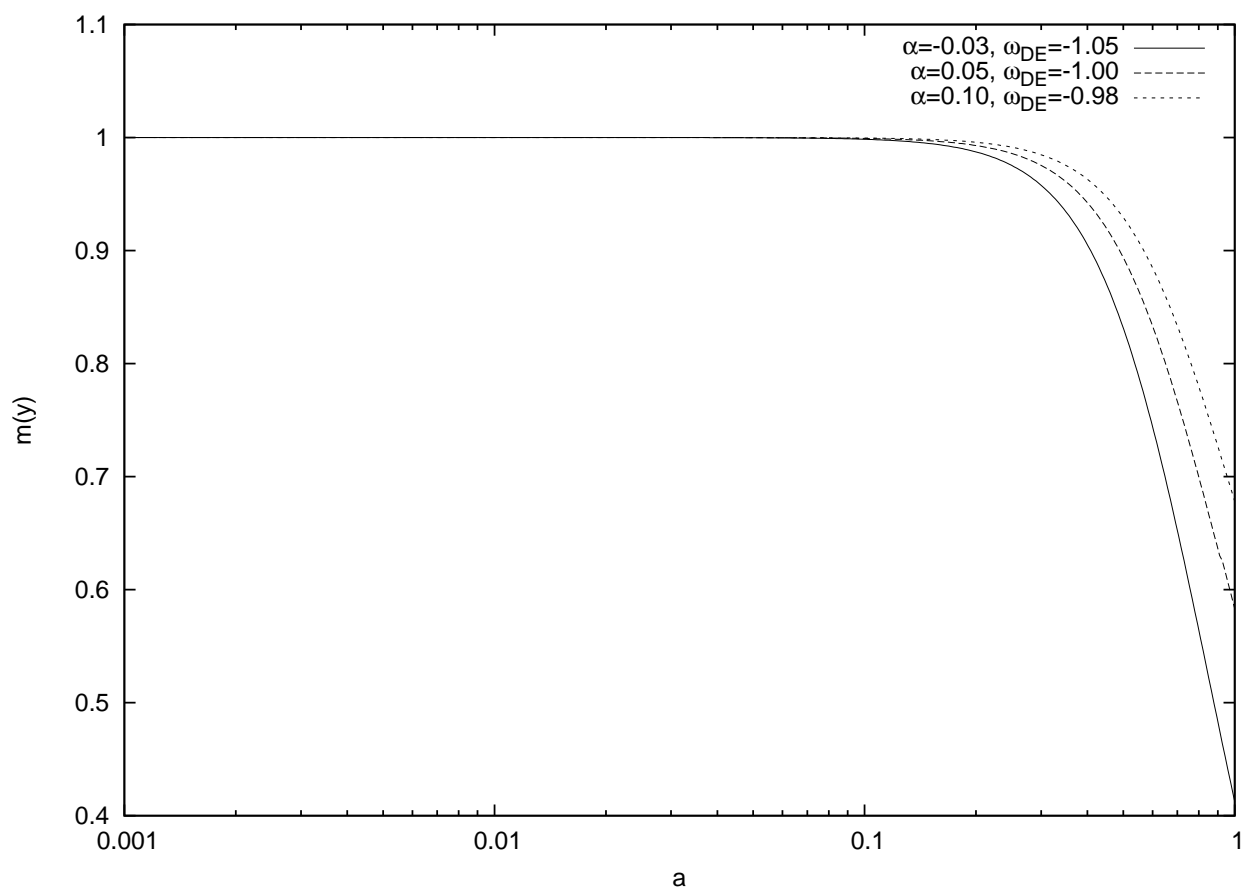

Figure 11. The growth factor $m(y)$ as a function of scale factor $a$. 


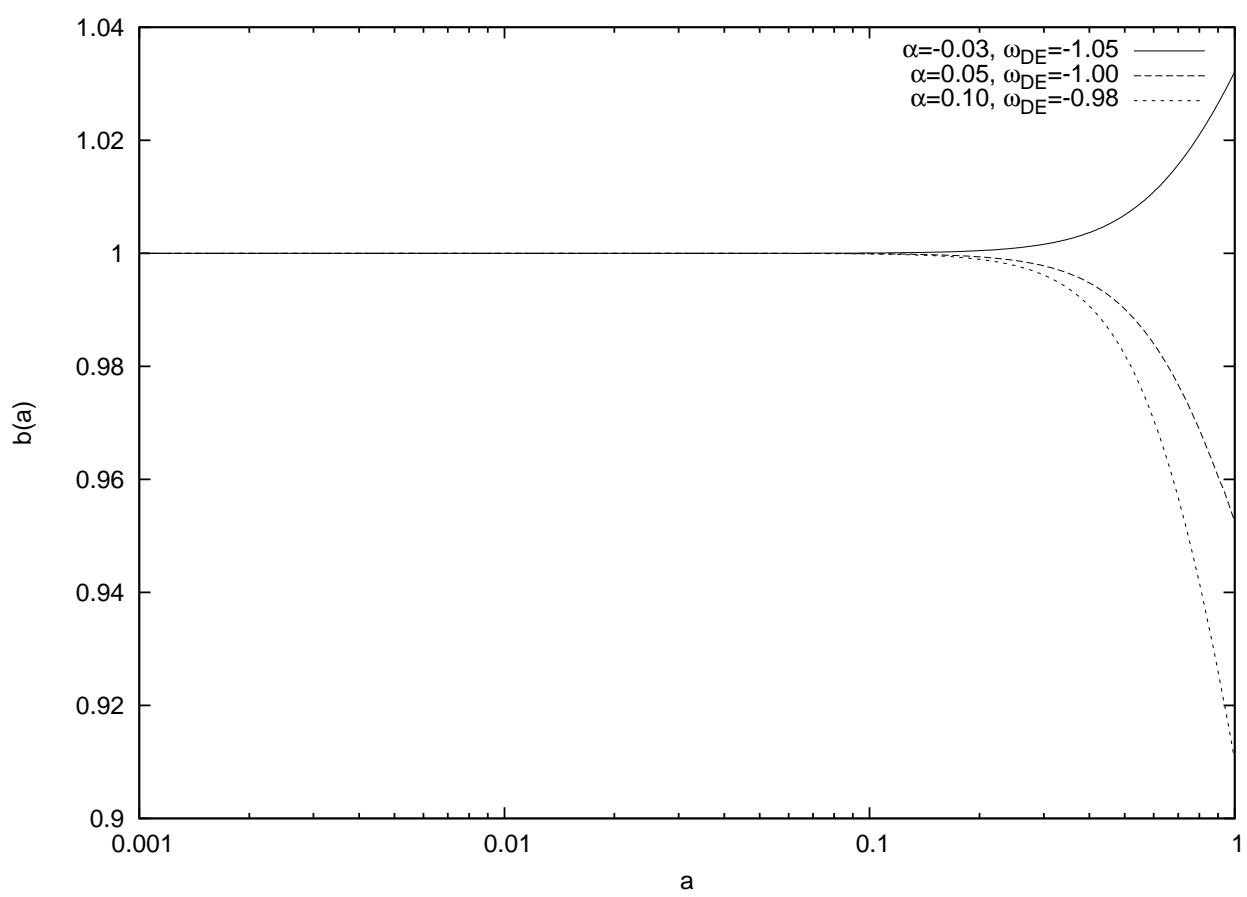

Figure 12. The bias $b(a)$ as a function of scale factor $a$.

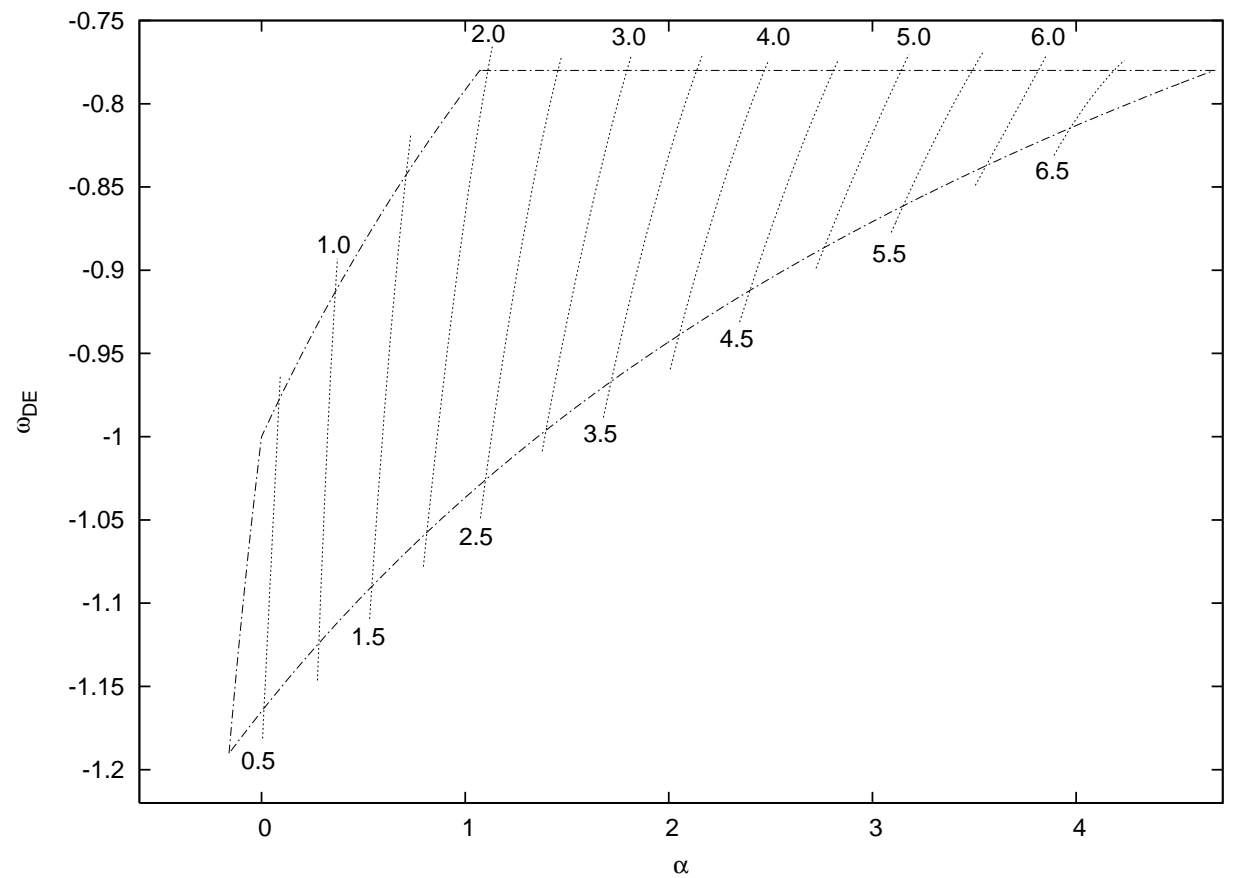

Figure 13. The contours for growth factor $m$ in the $\alpha-\omega_{D E}$ plane. 


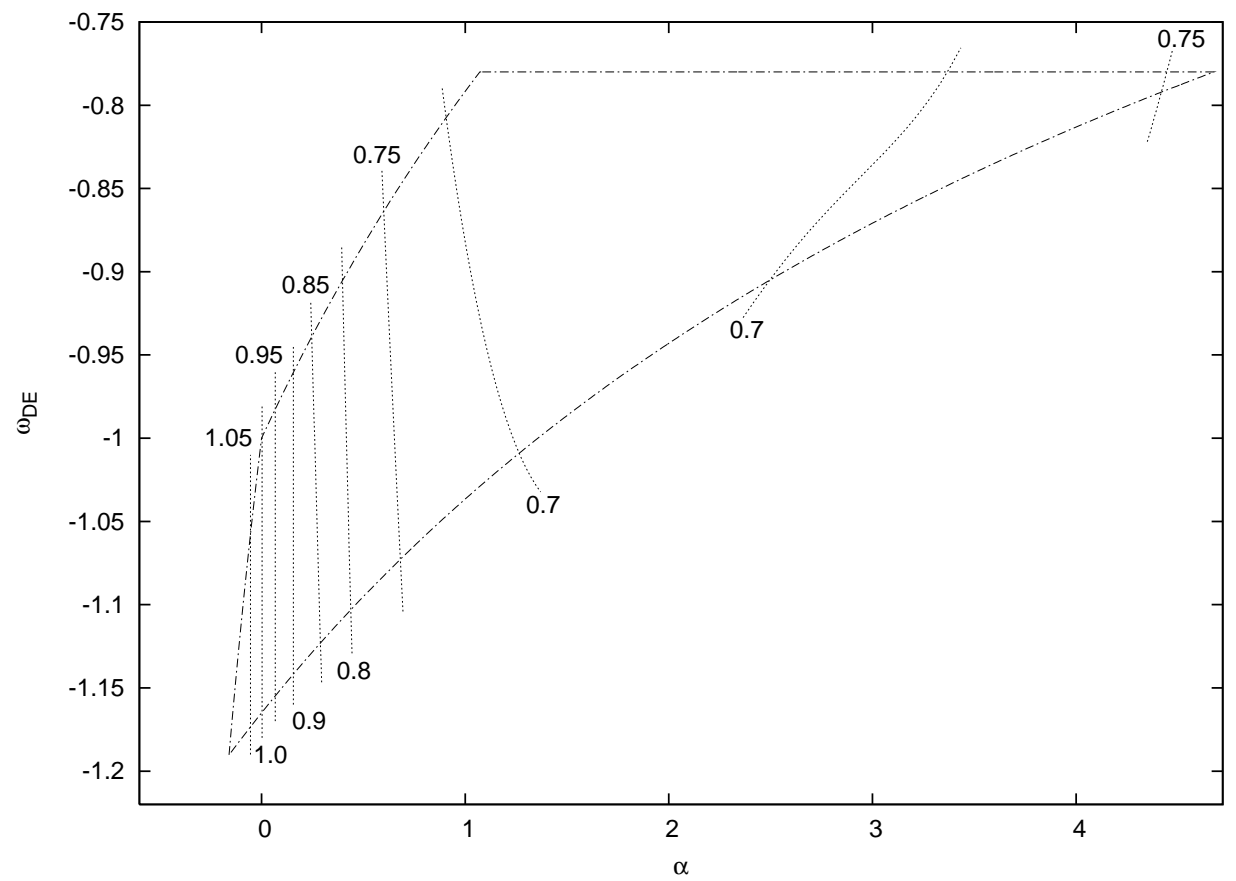

Figure 14. The contours for bias $b$ in the $\omega_{D E}-\alpha$ plane.

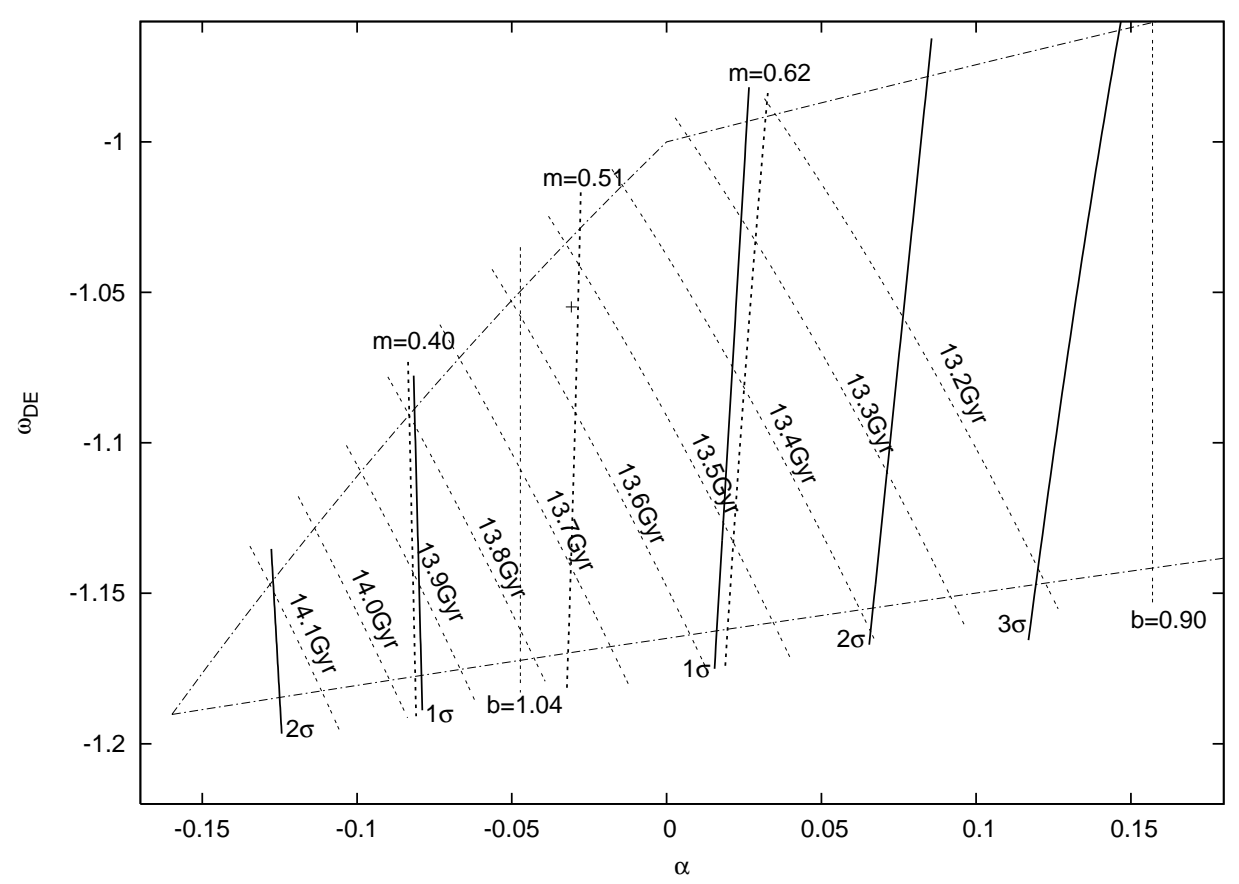

Figure 15. The contours in solid lines come from four limits of $c_{s}^{2}, t_{0}, m$ and $b$ in the $\omega_{D E}-\alpha$ plane. The confidence level contours of $68.3 \%, 95.4 \%$ and $99.73 \%$. The $1 \sigma$ fit values for our model parameters are $\alpha=-3.07_{-4.98}^{+5.66} \times 10^{-2}$ and $\omega_{D E}=-1.05_{-0.11}^{+0.06}$. And the plus sign in this figure denotes the best fits. 
velocity $\vec{v}$ are small, i.e., that $\delta_{D M} \ll 1$ and $\vec{v} \ll \vec{u}$, where $\vec{u}$ is the velocity in physical coordinate. Taking the divergence of Eq. (44) and substituting Eqs. (45) and (46), we finally obtain

$$
\begin{aligned}
& H^{2} \delta_{D M}^{\prime \prime}+\left(\dot{H}+2 H^{2}+H \frac{\Gamma}{\rho_{D M}}\right) \delta_{D M}^{\prime}-\left[4 \pi G \rho_{D M}\right. \\
& \left.-2 H \frac{\Gamma}{\rho_{D M}}-H\left(\frac{\Gamma}{\rho_{D M}}\right)^{\prime}\right] \delta_{D M}=0,
\end{aligned}
$$

where ${ }^{\prime} \equiv d / d \ln a$. Since the term $\frac{c_{s}^{2}}{a} \nabla \delta_{D M}$ is not included in the Euler equation, it leads to disappearance of the term $\nabla^{2} \delta_{D M}$ in Eq. (47). And we can easily see that there is no scale dependent term to drive oscillations or blowup in the power spectrum. We can solve Eq. (47) numerically, when the initial conditions are chosen as $a=10^{-3}$ and $\delta_{D M} \simeq 10^{-3}$, we plot the linear density perturbation for dark matter $\delta_{D M}$ as a function of $a$ (see Fig. 10). In Fig. 10, we can see the perturbation starts departing from the linear behavior around $a \simeq 0.80$ i.e. $z \simeq 0.25$ which is similar to the epoch when $z<z_{e q}$. Through the energy transferring from dark matter to dark energy, we can see that the dominance of dark energy is related to the time when the density fluctuations start deviating from the linear behavior.

When we consider the period after decoupling, the baryons are no longer coupled to photons so that the baryons are also a pressureless fluid like the dark matter. Then, we derive the baryon perturbation as follows

$$
H^{2} \delta_{B}^{\prime \prime}+\left(\dot{H}+2 H^{2}\right) \delta_{B}^{\prime}-4 \pi G \rho_{D M} \delta_{D M}=0 .
$$

Then we investigate the growth factor $m(y)$ and the bias $b(a)$ where $m(y)=D^{\prime}(y) / D(y)$, $D(y)$ is the linear growth function, $y=\ln (a)$ and $b=\delta_{B} / \delta_{D M}$. One can see from Fig. 11 and Fig. 12 that between the present and $z \simeq 5$, the growth factor $m(y)$ and the bias $b(a)$ are quite sensitive to the values of $\alpha$ and $\omega_{D E}$. Subsequently, we consider the constraints from $m(y)$ and $b(a)$ on the $\alpha-\omega_{D E}$ plane. In Fig. 13 and Fig. 14, we have shown the contours for the growth factor $m(y)$ and the bias $b(a)$ in the $\alpha-\omega_{D E}$ plane under the boundary of domain constrained by $c_{s}^{2}$. On the other hand, the growth factor and the bias parameter at $z \simeq 0.15$ have been determined using the $2 \mathrm{dFGRS}$. The redshift space distortion parameter $\beta=0.49 \pm 0.09$, and the bias $b=1.04 \pm 0.11[35]$. For $\beta=m / b$, we can subsequently determine the constraint on the growth factor $m$ as $m=0.51 \pm 0.11$. Noted that the fitted values of $b$ and $\beta$ are sensitive to the underlying model and the observational constraints on them are obtained under $\Lambda \mathrm{CDM}$ when converting redshift to distances for the power spectra. The right way is to conduct data processing for $b$ and $\beta$ by using 2dFGRS data under our model. Then we can constrain $\alpha$ and $\omega_{D E}$ by using the fitted values. In this paper we use the above fitted data temporarily and our future work will focus on this tough work.

In Fig. 15, we consider four restrictions together to give the best estimation of $\alpha$ and $\omega_{D E}$. Firstly, we plot contours constraint imposed by $t_{0}$ (Fig. 9), $m$ (Fig. 13) and $b$ (Fig. 14) under the boundary of domain constrained by the sound speed. Each set leads to a reduced $\chi^{2}: \chi^{2}\left(\alpha, \omega_{D E}\right)=(\text { Theory values-Observation values })^{2} /($ Observational 
error $)^{2}$. To combine the constraints on $\alpha$ and $\omega_{D E}$ coming from the age of the universe $t_{0}$, growth factor $m$ and the bias $b$ within the domain given by the limits of the sound speed $c_{s}^{2}$, we have added their individual $\chi^{2}$ as if they were part of a total experiment with uncorrelated Gaussian errors: $\chi_{\text {total }}^{2}\left(\alpha, \omega_{D E}\right)=\chi_{t_{0}}^{2}\left(\alpha, \omega_{D E}\right)+\chi_{m}^{2}\left(\alpha, \omega_{D E}\right)+\chi_{b}^{2}\left(\alpha, \omega_{D E}\right)$. Therefore, we plot the contour level $\Delta \chi_{\text {total }}^{2}\left(\alpha, \omega_{D E}\right)=2.3, \Delta \chi_{\text {total }}^{2}\left(\alpha, \omega_{D E}\right)=6.17$ and $\Delta \chi_{\text {total }}^{2}\left(\alpha, \omega_{D E}\right)=11.8$, where $\Delta \chi_{\text {total }}^{2}\left(\alpha, \omega_{D E}\right)=\chi^{2}\left(\alpha, \omega_{D E}\right)-\left(\chi^{2}\left(\alpha, \omega_{D E}\right)\right)_{\min }$, defines, respectively, for two degrees of freedom the $68.3 \%, 95.4 \%$ and $99.73 \%$ confidence levels represented in Fig. 15. The $1 \sigma$ fit values are $\alpha=-3.07_{-4.98}^{+5.66} \times 10^{-2}$ and $\omega_{D E}=-1.05_{-0.11}^{+0.06}$. Noted that the presence of the cutoff contours' curves in Fig. 15 is due to the restricted area boundary which comes from the limits of $c_{s}^{2}$.

\section{Conclusion}

A new model named as the modified generalized Chaplygin gas (MGCG), which is a further generalization of the generalized Chaplygin gas, has been proposed in the present paper. As a version of the unified dark matter and dark energy, this MGCG fluid is consisted of dark matter and quiessence dark energy with constant $\omega_{D E}$. Firstly, fundamental cosmology equations for the MGCG have been described. For tests with future deep observations, we then consider the Statefinder diagnostic since it can probe the expansion dynamics of the universe through higher derivatives of the scale factor. Furthermore, we investigate the evolution of density perturbations and the structure formation in our model. Then, with applied the age of the universe data $t_{0}$, growth factor $m$ and the bias $b$ within the domain given by the limits of $c_{s}^{2}$, the parameters $\alpha$ and $\omega_{D E}$ are constrained: $\alpha=-3.07_{-4.98}^{+5.66} \times 10^{-2}$ and $\omega_{D E}=-1.05_{-0.11}^{+0.06}$. It has been shown that there are a little difference between our model and others. To determine whether our model could be a candidate model of dark energy, the analysis and discussion of the matter power spectrum for MGCG must be done. This is a subject of our future research.

\section{Acknowledgments}

X.-M. Deng thanks the suggestions and comments from a referee, which plays an important role for the improvement of this article. X.-M. Deng appreciates the support from the group of Almanac and Astronomical Reference Systems in the Purple Mountain Observatory of China.

\section{References}

[1] Riess, A. G. et al., Astron. J.116, 1009 (1998); Perlmutter, S. et al., Astrophys. J.517, 565 (2003); Tonry, J. L. et al., Astrophys. J.594, 1 (2003).

[2] Spergel, D. N. et al., Astrophys. J. Suppl. 148, 175 (2003);Bennett, C. L. et al., Astrophys. J. Suppl. 148, 1 (2003).

[3] Tegmark, M. et al, Astrophys. J.606, 702 (2004).

[4] Weinberg, S., Rev. Mod. Phys. 61, 1 (1989). 
[5] Peebles, P. J. E. and Ratra, B., Rev. Mod. Phys. 75, 559 (2003).

[6] Ratra, B. and Peebles, P. J. E., Phys. Rev. D 37, 3406 (1998); Caldwell, R. R., Dave, R., and Steinhardt, P. J., Phys. Rev. Lett. 80, 1582 (1998); Sami, M. and Padmanabhan, T., Phys. Rev. D 67, 083509 (2003).

[7] Armendariz-Picon, C., Mukhanov, V., and Steinhardt, P. J., Phys. Rev. D 63, 103510 (2001); Chiba, T., Phys. Rev. D 66, 063514 (2002); Scherrer, R. J., Phys. Rev. Lett. 93, 011301 (2004).

[8] Sen, A., J. High Energy Phys. 04, 048 (2002); Sen, A. J. High Energy Phys. 07, 065 (2002); Gibbons, G. W. Phys. Lett. B 537, 1 (2002).

[9] Caldwell, R. R., Phys. Lett. B 545, 23 (2002); Elizalde, E., Nojiri, S., and Odintsov, S. D. Phys. Rev. D 70, 043539 (2004); Cline, J. M., Jeon, S., and Moore, G. D. Phys. Rev. D 70, 043543 (2004).

[10] Feng, B., Li, M., Piao, Y., and Zhang, X., Phys. Lett. B 634, 101 (2006); Guo, Z., Piao, Y., Zhang, X., and Zhang, Y., Phys. Rev. D 74, 127304 (2006).

[11] Kachru, S., Schulz, M., and Silverstein, E. Phys. Rev. D 62, 045021 (2000); Carroll, S. M., and Mersini, L., Phys. Rev. D 64, 124008 (2001); Kachru, S., Kallosh, R., Linde, A., and Trivedi, S. P., Phys. Rev. D 68, 046005 (2003).

[12] Carroll, S., et al, Phys. Rev. D 70, 043528 (2004); Deffayet, C., Dvali, G., and Gabadadze, G. Phys. Rev. D 65, 044023 (2002).

[13] Bento, M. C., Bertolami, O., and Sen, A. A., Phys. Rev. D 66, 043507 (2002); Bento, M. C. , Bertolami, O., and Sen, A. A., Phys. Rev. D 67, 063003 (2003); Bento, M. C., Bertolami, O., and Sen A. A., Phys. Rev. D 70, 083519 (2004); Bento, M. C., Bertolami, O., Santos, N. M. C., and Sen, A. A., Phys. Rev. D 71, 063501 (2005); Sen, A. A., and Scherrer, R. J., Phys. Rev. D 72, 063511 (2005); Banerjee, R., Ghosh, S., and Kulkarni, S., Phys. Rev. D 75, 025008 (2007).

[14] Bouhmadi-López, M., and Moniz, P. V., Phys. Rev. D 71, 063521 (2005); Giannantonio, T., and Melchiorri, A., Class. Quant. Grav. 23, 4125 (2006); Monerat, G. A., et al, Phys. Rev. D 76, 024017 (2007); Heydari-Fard, M., and Sepangi, H. R., Phys. Rev. D 76, 104009 (2007).

[15] Silva, P. T., and Bertolami, O., Astrophys. J. 599, 829 (2003); Bertolami, O., Sen, A. A., Sen, S., and Silva, P. T., Mon. Not. R. Astron. Soc. 353, 329 (2004).

[16] Kamenshchik, A., Moschella, U., and Pasquier, V., Phys. Lett. B 511, 265 (2001).

17] Sandvik, H., Tegmark, M., Zaldarriaga, M., and Waga, I., Phys. Rev. D 69, 123524 (2004); Sahni, V., Lect. Notes Phys. 653, 141 (2004).

[18] Copeland, E. J., Sami, M., and Tsujikawa, S., Int. J. Mod. Phys. D 15, 1753 (2006).

[19] Chimento, L. P., Phys. Rev. D 69, 123517 (2004); Chimento, L. P., and Lazkoz, R., Class. Quant. Grav. 23, 3195 (2006).

[20] Zhang, X., Wu, F. Q., and Zhang, J., J. Cosmol. Astropart. Phys. 01003 (2006).

[21] Knop, R. A., et al, Astrophys. J. 598, 102 (2003); Riess, A. G., et al, Astrophys. J. 607, 665 (2004).

[22] Barrow, J. D., Bean, R., and Magneijo, J., Mon. Not. R. Astron. Soc. 316, 41 (2000); Tegmark, M., et al, Phys. Rev. D 69, 103501 (2004)

[23] Sahni, V., Saini, T. D., Starobinsky, A. A., and Alam, U., JETP Lett. 77, 201 (2003); Alam, U., Sahni, V., Saini, T. D., and Starobinsky, A. A., Mon. Not. R. Astron. Soc. 344, 1057 (2003).

[24] Jarosik, N., et al., Astrophys. J. Suppl. 192, 14 (2011).

25] Colless, M., et al., Mon. Not. R. Astron. Soc. 328, 1039 (2001).

[26] Podariu, S., Daly, R. A., Mory, M. P., and Ratra, B., Astrophys. J. 584, 577 (2003)

[27] Gorini, V., Kamenshchik, A. Y., Moschella, U., Pisttella, O. F., and Starobinsky, A. A., J. Cosmol. Astropart. Phys. 02, 016 (2008)

[28] Gorini, V., Kamenshchik, A., and Moschella, U., Phys. Rev. D 67, 063509 (2003); Zimdahl, W., and Pavon, D., Gen. Rel. Grav. 36, 1483 (2004).

[29] Aldering, G., et al, SPIE 4835 146A (2002).

[30] Arcuri, R. C., and Waga, I., Phys. Rev. D 502928 (1994).

[31] Mukhanov, V., Physical Foundations of Cosmology, (Cambridge university press, 2005). 
[32] Jimenez, R., Thejll, P., Jorgensen, U., MacDonald, J., and Pagel, B., Mon. Not. R. Astron. Soc. 282, 926 (1996).

[33] Richer, H., et al, Astrophys. J. 574 L151 (2002).

34] Hansen, B., et al, Astrophys. J. 574, L155 (2002).

[35] Verde, L., et al, Mon. Not. R. Astron. Soc. 335432 (2002); Hawkins, E., et al, Mon. Not. R. Astron. Soc. 34678 (2003); Linder, E. V., arXiv:0709.1113 (2007). 